\title{
Precise revolution control in three-dimensional off-axis trapping with single Laguerre-Gaussian beam
}

\author{
Tomoko Otsu • Taro Ando • Yu Takiguchi • \\ Yoshiyuki Ohtake $\cdot$ Haruyoshi Toyoda · \\ Hiroyasu Itoh
}

Received: 16 June 2014/Accepted: 2 September 2014/Published online: 25 February 2015

(c) The Author(s) 2015. This article is published with open access at Springerlink.com

\begin{abstract}
We report the precise study of orbital angular momentum transfer from Laguerre-Gaussian (LG) beams to submicrometer-sized dielectric spheres. Stable three-dimensional off-axis trapping of the spheres was achieved and confirmed by the constant behavior of the sphere's revolution radius against the trapping LG-beam power. By measuring the revolutions of the sphere that was trapped and revolved in mid-water, we confirmed excellent linearity between the revolution rate and the trapping light free from the hydrodynamic coupling with walls of a sample chamber. This result suggests a torque source of precise controllability, which will contribute to the study of biological molecules, nonequilibrium statistical physics, and micromachines.
\end{abstract}

Keywords Holographic optical tweezers - Optical vortices · Phase only spatial light modulator

Optical vortices (OVs) are known as a series of light beams with singular wavefronts. Due to their phase property, OVs carry orbital angular momenta around their beam axes to have an ability of revolving objects under light irradiation $[1,2]$. This suggests the possibility of maintaining the objects' revolutions by continuous irradiation of OVs without any dynamical control. Such revolutions have been achieved two-dimensionally on cover glasses [3-5] except for a few studies reporting three-dimensional revolution in

T. Otsu $(\bowtie) \cdot$ T. Ando · Y. Takiguchi · Y. Ohtake · H. Toyoda Central Research Laboratory, Hamamatsu Photonics K.K,

Hamamatsu, Shizuoka 434-8601, Japan

e-mail: t-otsu@crl.hpk.co.jp

H. Itoh

Tsukuba Research Center, Central Research Laboratory,

Hamamatsu Photonics K.K, Tsukuba, Ibaraki 300-2635, Japan mid-water with the help of gravity [6] or an additional Gaussian beam [7] for fixing the axial trapping positions. Whether a single OV can achieve three-dimensional trapping in cooperation with object's revolution remains unclear, but the authors [8] recently established direct evidence of three-dimensional trapping by LaguerreGaussian (LG) beams that are the most common embodiments of OVs.

In this report, we study the controllability of the revolution of micrometer-sized polystyrene spheres trapped by LG beams in mid-water. The trapping LG beams were generated by a holographic phase-amplitude modulation scheme to obtain high-quality ones [9]. To suppress the interaction of the trapped spheres with walls caused by Brownian motion in the axial direction, we observed the revolution of polystyrene spheres trapped in mid-water more than $1.5 \mu \mathrm{m}$ away from the surface of a cover glass. Measurements of revolution speed with varying LG-beam power revealed precise controllability of the revolutions by the trapping light power, suggesting a novel optical tool that is especially useful for investigating the torsional properties of biological molecules [10] and driving rotational micromachines [11].

Figure 1 schematically shows the present experimental setup. The linearly-polarized laser output (wavelength $\lambda=1,064 \mathrm{~nm}$ ) was expanded by a beam expander and shaped into a top-hat intensity profile through an aperture. The trapping LG beams were obtained by converting the top-hat light with holographic phase patterns displayed on a liquid-crystal-on-silicon spatial light modulator (LCOSSLM). The holographic patterns were prepared by a simultaneous phase-amplitude modulation method to generate high-quality LG beams [9] of arbitrary orders. The basic concept of the phase-amplitude modulation method is to superimpose a desired phase profile onto a blazed phase- 


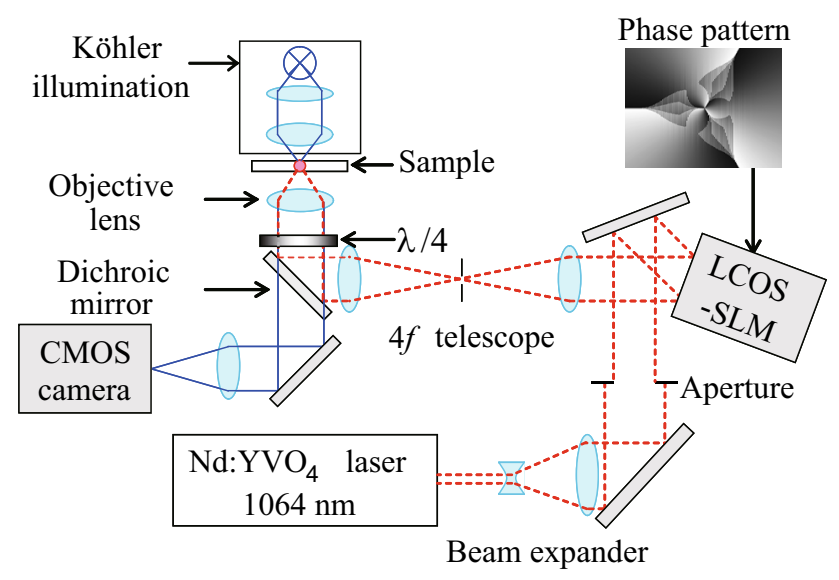

Fig. 1 (Color online) Experimental setup. Dashed lines from laser source to sample indicate light path of a trapping LG beam, while solid lines from illumination source to CMOS camera indicate that of observed images.

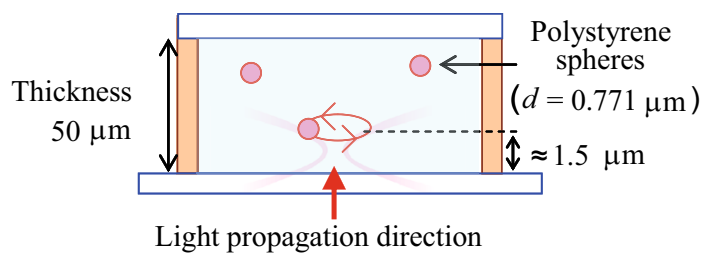

Fig. 2 (Color online) Sample structure. Polystyrene spheres of $0.771-\mu \mathrm{m}$-diameter were infused in ultra-pure water placed between a pair of cover glasses separated by $50 \mu \mathrm{m}$.

grating pattern whose blazing amplitudes were locally modulated with respect to a desired amplitude profile. In the present experiments, the holographic phase-amplitude modulation patterns were prepared based on 4-level blazed phase grating. Resultantly, We obtained high-quality LG beams of azimuthally second, third, and fourth orders as the first-order diffraction components from the holographic patterns. The generated LG beams were transferred by a $4 f$ telescope to the back aperture of an oil-immersion objective lens $(\mathrm{NA}=1.30)$ through a dichroic mirror. The focused LG beams were applied to a sample after being converted to circular polarization with a quarter-wave plate placed at the back aperture. The trapping LG beams were converted to circular polarization to keep the circular focusing patterns even under tight-focusing conditions. The sample was prepared by suspending polystyrene spheres (diameter $d=0.771 \mu \mathrm{m}$ ) in ultra-pure water and putting them between a pair of cover glasses separated by $50-\mu \mathrm{m}-$ thick spacers (Fig. 2).

We acquired the images of the trapped spheres under visible-light illumination using a high-speed CMOS camera to record the revolution of the spheres. The images of the revolving polystyrene spheres (Fig. 3a) were converted to a time series of centroid information (Fig. 3b) that was (a)

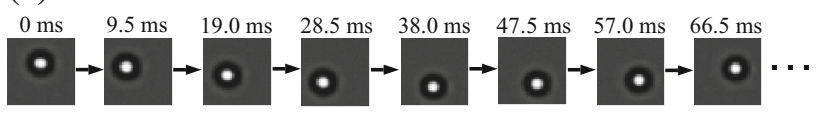

(b)

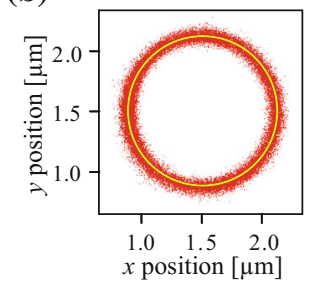

(c)

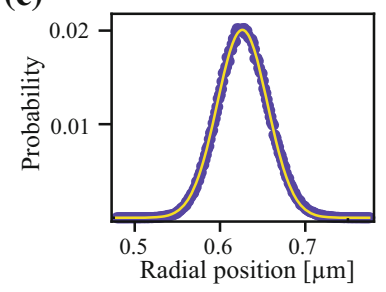

Fig. 3 (Color online) Data analysis flow. a Sampled images of a revolving polystyrene sphere observed by CMOS camera. Practically, images were observed at $6,335 \mathrm{~Hz}$. b Centroid-position distribution derived from images. c Histogram of radial-position distribution.

calculated by a center-of-mass algorithm. The centroids can be determined with a position uncertainty of less than 0.01 pixel corresponding to $1 \mathrm{~nm}$ under the present setup. The center of the revolution was determined by fitting a series of the centroid data to a circular orbit, and the radialposition distribution was obtained with respect to the center. Finally, we obtained revolution radius $R_{\mathrm{s}}$ was derived by fitting the radial distribution to a Gaussian profile (Fig. 3c), and average revolution rate $f$ was also determined from the change in the azimuthal position per unit time.

We observed the changes in $R_{\mathrm{S}}$ versus trapping LGbeam power $P_{\mathrm{LG}}$ under various conditions that were determined from combinations of the polarization direction and azimuthal-mode index $l(=2,3$, and 4$)$ of the LG beams. Polystyrene spheres were trapped around the position separated by about $1.5 \mu \mathrm{m}$ from the front (lower) cover glass[8] (Fig. 2), and the $P_{\mathrm{LG}}$ values were measured at the sample position under a dry condition. We roughly evaluated gravity's contribution to the trapping by the following logic. First, the LG beams are assumed to propagate with keeping nearly similar beam patterns with different sizes, meaning that a cross-sectional light-power density should be inversely proportional to the square of the beam radius. Based on this assumption, note that the revolution radius increases when $P_{\mathrm{LG}}$ becomes larger in the case of the trapping achieved under the balance of the light's scattering force and the gravity on the sphere. Thus $R_{\mathrm{S}}$ will be proportional to a square root of $P_{\mathrm{LG}}$ for gravitysupported trapping, while the radius is independent of $P_{\mathrm{LG}}$ for gradient-force trapping, i.e., trapping due only to an action of light. Figure 4 shows the relationship between $R_{\mathrm{S}}$ and $P_{\mathrm{LG}}$ for $l=2,3$, and 4 , where the solid and dashed lines are the best-fitted linear models for the measured $R_{\mathrm{S}}$ values. The changes in $R_{\mathrm{S}}$ against $P_{\mathrm{LG}}$ are almost negligible in all cases; in other words, the behavior of $R_{\mathrm{S}}$ does not 


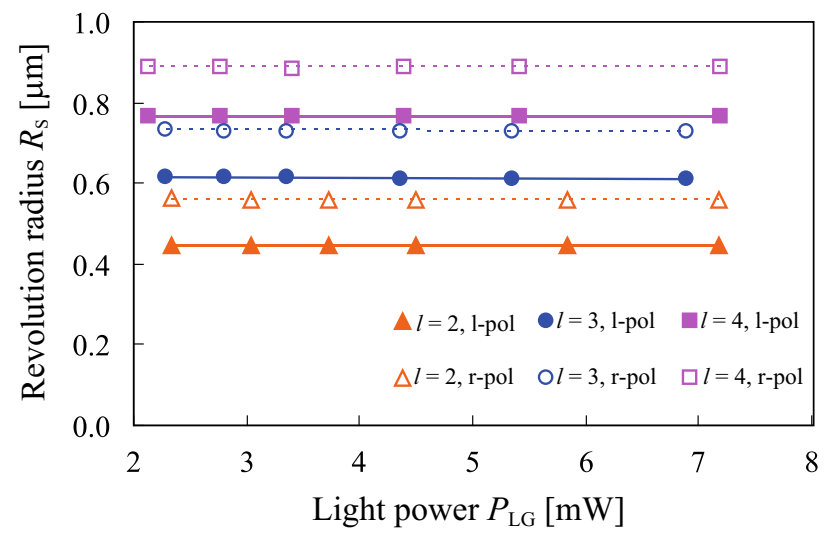

Fig. 4 (Color online) Behavior of revolution radii $R_{\mathrm{s}}$ of a trapped polystyrene sphere with respect to changes of trapping LG-beam power $P_{\mathrm{LG}}$. Lines show best-fitted linear models for the behavior. Triangle, circle, and square symbols are results for $l=2,3$, and 4 LG beams, respectively, while closed symbols with solid lines and open symbols with dotted lines correspond to the results for leftpolarized (l-pol) and right-polarized (r-pol) LG beams, respectively.

match the square-root models predicted for gravity-assisted trapping. This result indicates that optical trapping by LG beams is almost achieved due to the balance of the scattering and gradient forces of light without the help of gravity.

Note also that $R_{\mathrm{S}} \mathrm{s}$ for the right-polarized (r-pol) LG beams are always larger than those for the left-polarized (1pol) ones in Fig. 4. This is consistent with the behavior of the LG beams focused tightly by a high numerical aperture objective lens [12-14]. In other words, the change in $R_{\mathrm{s}}$ due to the circular polarization direction of the LG beams is additional evidence for proper trapping on the bright rings of the focused LG beams.

Figure 5 shows the relationship between rotation rate $f$ and LG-beam power $P_{\mathrm{LG}}$. The revolution rates are proportional to the LG-beam power, suggesting the almost complete controllability of the object's revolution with incident LG-beam power. To further discuss the change in $f$ for the LG-beam polarization, recall that orbital angular momentum $J$ of the revolving sphere (mass $m$ ) is given as

$$
J=2 \pi f m R_{\mathrm{s}}{ }^{2} .
$$

Table 1 exhibits ratio $J_{\mathrm{r}-\mathrm{pol}} / J_{1-\text { pol }}$ between the orbital angular momenta induced by the left- and right-polarized trapping LG beams for $l=2,3$, and 4 . Here, each $J_{\mathrm{r}-\text { pol }} / J_{1-\text { pol }}$ was derived from the fitting results of $R_{\mathrm{S}}$ and the revolution speed in Figs. 4 and 5. The present experiments were performed for non-absorptive and nonbirefringent dielectric spheres, which receive no "spin" angular momenta from the polarization component of light. Hence, the polarization of the trapping light is expected to be independent of the objects' revolution; i.e., $J_{\mathrm{r}-\mathrm{pol}} / J_{1-\text { pol }}$ should become unity.This deviation in the experimental
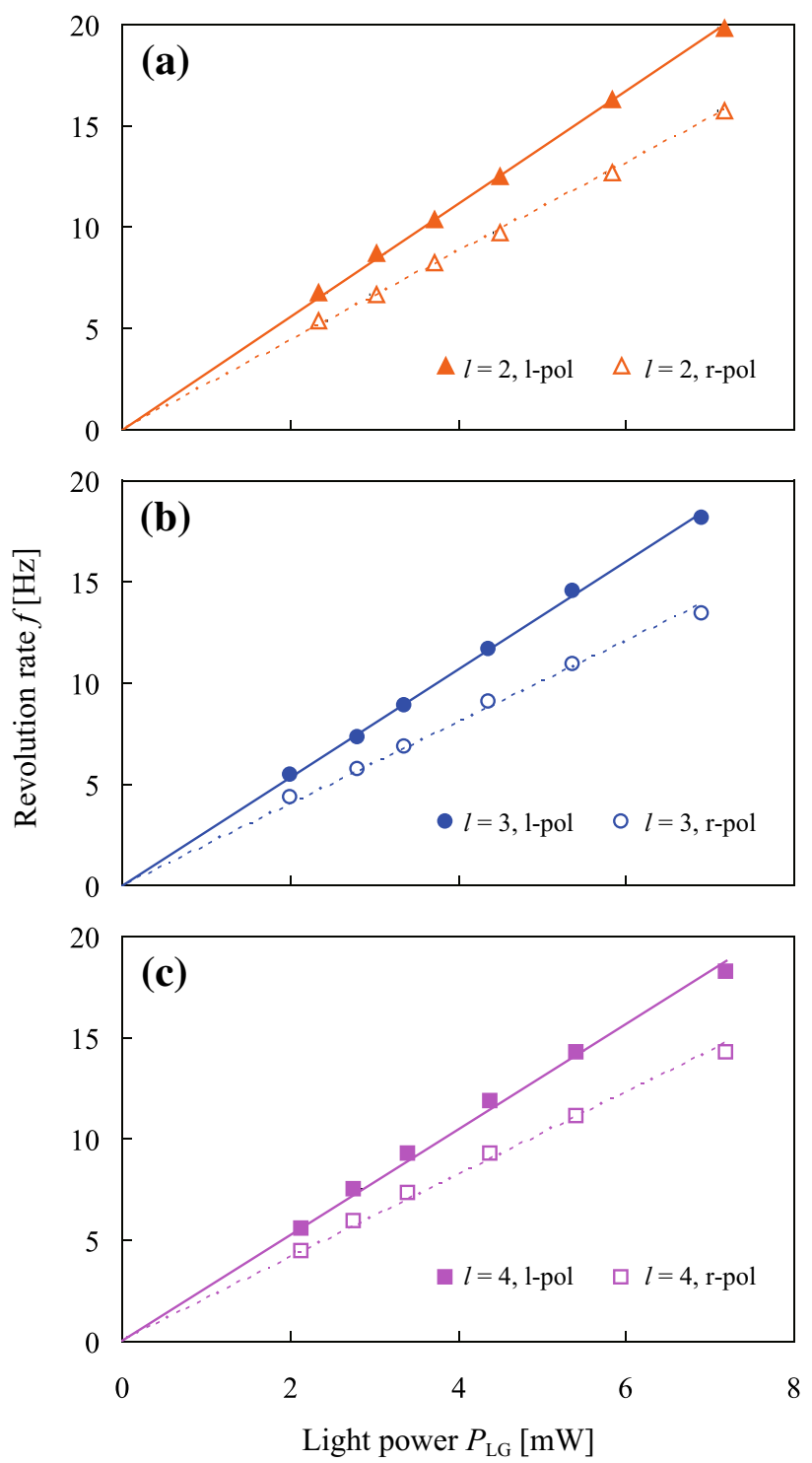

Fig. 5 (Color online) Revolution rate $f$ versus LG-beam power $P_{\mathrm{LG}}$ for $\mathbf{a} l=2$, b $l=3$, and $\mathbf{c} l=4 \mathrm{LG}$ beams. Meanings of symbols and lines resemble those in Fig. 4.

Table 1 Ratio between orbital angular momenta induced by left- and right-polarized trapping LG beams for $l=2,3$, and 4 derived from results in Figs. 4 and 5

\begin{tabular}{llll}
\hline & $l$ & & \\
\cline { 2 - 4 } & 2 & 3 & 4 \\
\hline$J_{\mathrm{r}-\text { pol }} / J_{1-\text { pol }}$ & 0.80 & 0.94 & 0.95 \\
\hline
\end{tabular}

results from the above perspective also relates to the polarization-dependent deformation of a tightly focused LGbeam pattern [12-14]. Here, non-paraxial solutions of the Helmholtz equation [13-15] are suitable for discussing the angular-momentum properties of tightly focused circular- 
symmetric LG beams. According to the non-paraxial description, the mechanism of focal-pattern deformation can be explained in principal as follows; the LG patterns due to the lateral components of the light amplitude are independent of polarization direction, and the deformation is caused mainly by the axial light-amplitude component. We consider that the axial light-amplitude component varies $R_{\mathrm{S}}$ to change the net orbital angular-momentum transfer from the lateral light-amplitude components to the trapped object, resulting in the non-unity values of $J_{\mathrm{r}-\text { pol }} / J_{1-\text { pol }}$ in Table 1. In other words, this phenomenon is an example of the coupling effects between the optical spin and orbital angular momenta in the tight-focusing regime [14, 15], which should be of future interest regarding the mechanical property of tightly focused light.

In summary, we reported the precise revolution control of micrometer-sized polystyrene spheres trapped by LG beams in mid-water. We examined the behavior of the revolution radius against trapping LG-beam power under conditions where the support of a glass surface lies too far from trapped objects to affect the trapping position in the light propagation direction. LG trapping was achieved in mid-water without the help of gravity. Furthermore, by measuring the revolution speed by varying the LG-beam power, we confirmed that the revolution rate is proportional to the LG-beam power. Based on the result, it is possible to precisely control objects' revolutions by changing the LGbeam power. The present technology of a highly controllable torque source will contribute to the study of biological molecules and micromachines.

Acknowledgments The authors are grateful to A. Hiruma (president), T. Hara (director), S. Ohsuka, and T. Inoue of Hamamatsu
Photonics for their encouragement throughout this work as well as to H. Takamoto, H. Ito, N. Matsumoto, and Y. Hashimoto for their helpful support of the microscope, the spatial light modulator unit, and the holographic setup.

Open Access This article is distributed under the terms of the Creative Commons Attribution License which permits any use, distribution, and reproduction in any medium, provided the original author(s) and the source are credited.

\section{References}

1. L. Allen, S.M. Barnett, M.J. Padgett, Optical angular momentum (IoP Publishing, Bristol, 2003)

2. D.G. Grier, Nature 424, 810 (2003)

3. V. Garcés-Chávez, K. Volke-Sepulveda, S. Chávez-Cerda, W. Sibbett, K. Dholakia, Phys. Rev. A 66, 063402 (2002)

4. V. Garcés-Chávez, D. McGloin, M.J. Padgett, W. Dultz, H. Schmitzer, K. Dholakia, Phys. Rev. Lett. 91, 093602 (2003)

5. M. Chen, M. Mazilu, Y. Arita, E.M. Wright, K. Dholakia, Opt. Lett. 38, 4919 (2013)

6. A.T. O’Neil, M.J. Padgett, Opt. Commun. 185, 139 (2000)

7. G. Volpe, D. Petrov, Phys. Rev. Lett. 97, 210603 (2006)

8. T. Otsu, T. Ando, Y. Takiguchi, Y. Ohtake, H. Toyoda, H. Itoh, Sci. Rep. 4, 4579 (2014)

9. T. Ando, Y. Ohtake, N. Matsumoto, T. Inoue, N. Fukuchi, Opt. Lett. 34, 34 (2009)

10. H. Itoh, A. Takahashi, K. Adachi, H. Noji, R. Yasuda, M. Yoshida, K. Kinosita Jr, Nature 427, 465 (2004)

11. S. Maruo, H. Inoue, Appl. Phys. Lett. 91, 84101 (2007)

12. Y. Iketaki, T. Watanabe, N. Bokor, M. Fujii, Opt. Lett. 32, 2357 (2007)

13. S.H. Simpson, S. Hanna, J. Opt. Soc. Am. A 26, 625 (2009)

14. S.H. Simpson, S. Hanna, J. Opt. Soc. Am. A 27, 2061 (2010)

15. S.M. Barnett, L. Allen, Opt. Commun. 110, 670 (1994) 